\title{
X-ray fluctuation timescale and Black Hole mass relation in $\mathrm{AGN}$
}

\author{
Amri Wandel ${ }^{1}$ and Mathew Malkan ${ }^{2}$ \\ ${ }^{1}$ Racah Institute, The Hebrew University of Jerusalem, \\ Jerusalem 91904, Israel \\ email: amri@huji.ac.il \\ ${ }^{2}$ Dept. of Astronomy, University of California, Los Angeles, California, U.S.A. \\ email: malkan@astro.ucla.edu
}

\begin{abstract}
We analyze the fluctuations in the X-ray flux of 20 AGN (mainly Seyfert 1 galaxies) monitored by RXTE and XMM-Newton with a sampling frequency ranging from hours to years, using structure function (SF) analysis. We derive SFs over four orders of magnitude in the time domain (0.03-300 days). Most objects show a characteristic time scale, where the SF flattens or changes slope. For 10 objects with published power-spectral density (PSD) the break time scales in the SF and PSD are similar and show a good correlation. We also find a significant correlation between the SF timescale and the mass of the central black hole, determined for most objects by reverberation mapping.
\end{abstract}

Keywords. AGN, X-ray, Fluctuations, Structure Functions, Power Spectral Density, Black Holes

\section{Introduction}

The strong, irregular X-ray variability observed in many Seyfert galaxies on timescales as short as hours indicates that these X-rays are emitted from a region close to the central black hole. Doubling time scales were used as the first quantification of variability; characteristic time scales of X-ray variability seemed to correlate with luminosity and black hole mass Wandel \& Mushotzky (1986).

For quasi-periodic and stochastic light curves the variability is often characterized through the power spectral density (PSD). The PSD is the variability amplitude per frequency, so it describes the variability power contained within a frequency interval. A similar measure that is sometimes used is the structure function, which describes the variability amplitude as a function of time scale. The variability of quasi-periodic and stochastic light curves may then be characterized by representing the power spectrum in a parametric form. However, irregular sampling or sequences of regular sampling separated by gaps often cause problems in measuring variability features from a light curve, which is the case in all ground-based and often also space-based data. Ideally the variability must be quantified over a broad a range of time scales, to maximize the probability of detecting a characteristic variability time scale and to avoid spurious features caused by an insufficiently long observing time.

The Rossi X-ray Timing Explorer (XTE) has been the first mission to provide sustained monitoring on given objects for time scales up to months and years. High dynamicrange X-ray PSDs were published for approximately 10 AGN, (e.g., Edelson \& Nandra (1999), Uttley, McHardy \& Papadakis (2002), Markowitz et al. (2003), Chatterjee et al. (2009;2011), Rothshild et al. (2011)). Typically they are parameterized as a broken power law; on long timescales the fluctuation power is flat, scaling as a power of $f^{0-1}$, and on short timescales it is descending more steeply, with slopes of 2-3. The break between 


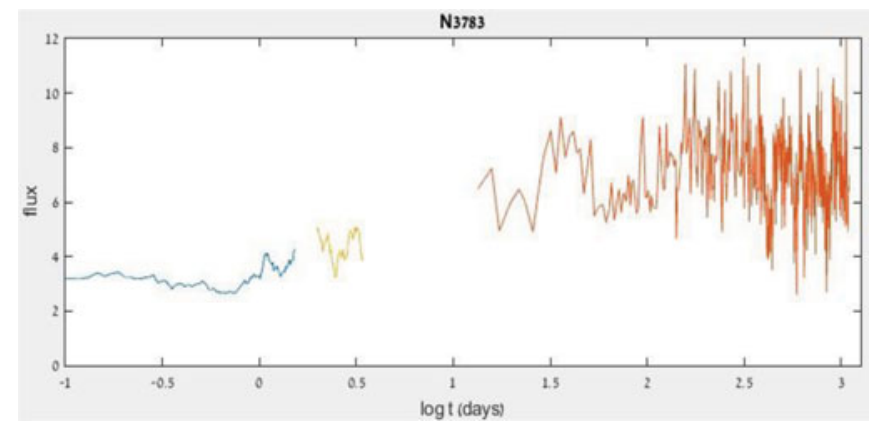

Figure 1. Combined logarithmic light curve of NGC 3783. The X-ray flux vs. log (1/frequency) in units of days. Shown are the light curves of XTE (right curve) and XMM (the two left curves).

these two regimes is in the range of roughly $10^{4-6} \mathrm{~Hz}$, corresponding to time scales $t_{P S D}$ ranging from a few hours up to weeks.

\section{Structure functions}

The structure function (SF) measures the mean relative amplitudes of pairs of flux variations as a function of their time difference (Simonetti et al. (1985)). While the PSD requires strictly even sampling to compute, the SF can be derived for uneven and gappy sampling. The SF has been applied to optical AGN monitoring data (Webb and Malkan (2000); Collier \& Peterson (2001)) as well as to X-ray light curves of blazars. As long as the number/size of gaps are not too large, meaningful results can be obtained; however this procedure is not foolproof, and sampling limitations (especially the lack of long enough timescales) can produce spurious features, as demonstrated by Emmanoulopoulos, McHardy \& Uttley (2010) (hereafter E10), who give a detailed analyses of the caveats and cautions for SF measurement and interpretation. Recently a new method - continuous-time autoregressive moving average (CARMA) has been suggested (Kelly et al. 2014) to handle irregular sampling and gaps.

We define the amplitude-averaged SF as

$$
S(\tau)^{2}=\sum_{k}\left[f\left(t_{k}\right)-f\left(t_{k}-\tau\right)\right]^{2}
$$

where $f\left(t_{k}\right)$ is the normalized flux at time point $t_{k}$. As advised in E10, we work with the $\log f$-log SF. Typically, the fluctuation amplitude is increasing with time difference, as larger fluctuations are present on longer timescales.

To derive errors on each SF bin, we used two methods. The first is an enhanced $\chi^{2}$ method (Collier \& Peterson (2001)). The statistical uncertainties in structure functions are defined by $\left.\sigma_{i}\right) /\left(N_{i} / 2\right)^{1 / 2}$ where $\sigma_{i}$ is the root mean square (rms) deviations about the mean structure function value in bin i and $N_{i}$ is the number of pairs of data points in the $i$-th bin. This ad hoc, but reasonable, error estimates reflect the fact that not all pairs of measurements in a given bin are independent. The second method, which for sparser sampling gives more realistic errors for light curves, is a Monte Carlo procedure (e.g., Kataoka et al. (2001) and E10).

For each light curve/SF segment we assume a form for the underlying PSD (using the published PSD parameters if they exist; otherwise we make a PSD analyses with the incomplete data and use the resulting power law (Emmanoulopoulos, private communication). For each curve segment, we simulate 100 light curves using the algorithm of Timmer \& Koenig (1995), re-sample them in the same manner as the observed light 

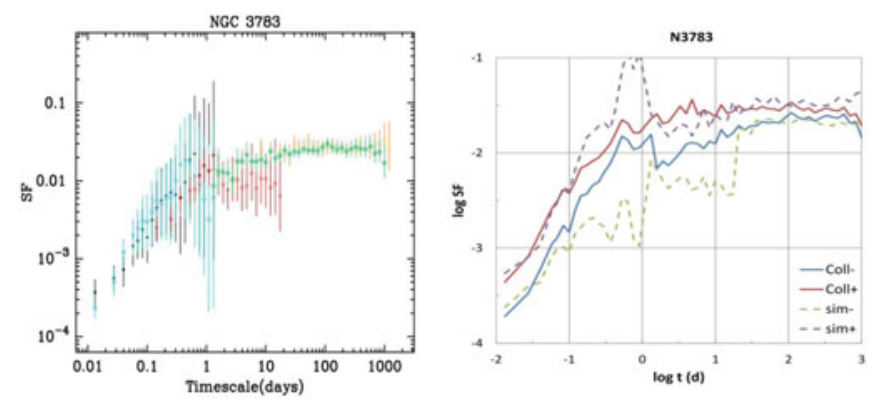

Figure 2. Combined $\log (\mathrm{SF})-\log \mathrm{t}$ diagram for NGC 3783.Upper and lower error limits, calculated for the $95 \%$-th percentile with both methods, MC simulations (dashed) and Collier \& Peterson (2001) (solid).

curve, and measured the SF. This yields relatively larger errors towards longer time scales, which reflects systematic uncertainties in the SF introduced by red-noise leak and/or by gaps in the sampling. We adopt the $95 \%$ confidence errors on each binned SF point, as a trade-off between $1 \sigma$ errors for $\chi^{2}$-fitting and a higher confidence level, that would reflect more closely the systematic SF behavior for each time scale bin. Finally we combine the $\log (\mathrm{SF})-\log \mathrm{t}$ diagrams for all data segments for each object to produce a combined SF over a broad range of timescales.

Similar SF figs of have been obtained for NGC 3516, 4051, 4151, 5584, 7469, IC4329a, MCG 6-30-15, Fairall 9, Mkn 766. The two last ones do not show an obvious break (Wandel, Markowitz and Malkan, in preparation). Comparing $\chi^{2}$-fits of the SF to an unbroken power-law and a broken one, we find that for almost all objects with a full range time series ( 4 orders of magnitude in temporal range) data sources, the improvement in a $\chi^{2}$ fit when including a break is statistically significant. It is important though to caution that the SF points are not all independent, and unlike the Monte Carlo method applied to PSDs, this method also does not take into account distortion effects such as aliasing or red-noise leak, which could introduce scatter into the determination of the break point $t_{S F}$.

\section{The SF-break timescale and the Black Hole mass relation}

It can be shown that for good enough data

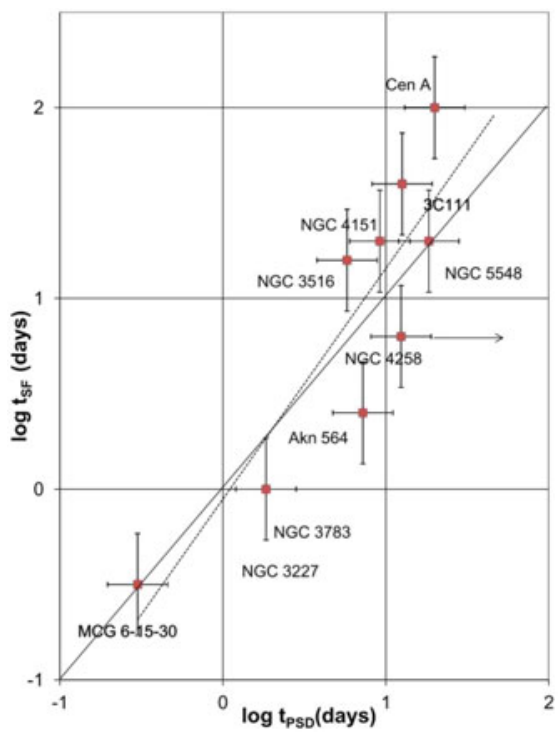

Figure 3. The time scale corresponding to the SF break vs. that of the break in the PSD. The best fit has a slope of 1. Solid line denotes equality, dashed line is the best fit. Typical errors are denoted by the crosses; the vertical error bars would be larger for a higher confidence level. the PSD and SF power-law slopes are related: when the sampling is of sufficient quality and dynamic range the slope of $\operatorname{PSD}(\nu)$ minus one equals the slope of $\operatorname{SF}(\tau)(\mathrm{E} 10$, appendix A). If a change in power-law slope exists in the broadband PSD, we should expect to find a change in power-law slope in the broadband SF at a similar time scale. Comparing the SF and the PSD timescales for the objects with published PSD, it appears that a distinct break in the SF corresponds to the break in the PSD. 


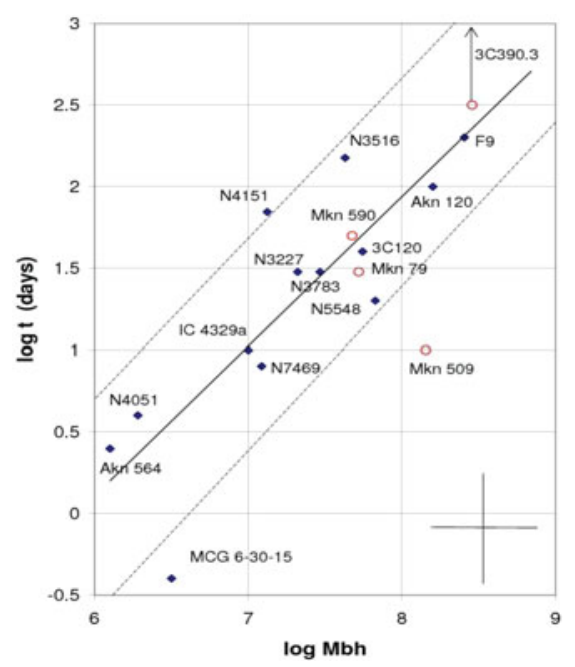

Figure 4. SF break time vs. black hole mass for 13 Seyfert galaxies. Four more objects with weak breaks are denoted by circles. The solid line is the best linear fit, dashed lines are linear extrapolations of the two breaks in the PSD of Cyg X-1. Typical errors are denoted by the cross in the lower right.
The PSD timescales scale approximately linearly with the estimated black hole mass (McHardy et al. (2006); Uttley and McHardy (2005)). Using the structure function method enables us to include also objects which do not have sufficient sampling for constructing a PSD. Altogether we have 17 objects. We find a similar relation between the SF break timescale and the BH mass (fig. 3). The black hole masses of most of the Seyferts are estimated by reverberation mapping (Peterson et al. 2004 ). For Akn 564, 3C111 and MCG 6-15-30, which do not have reverberation data, single-epoch $\mathrm{H} \beta$ is used with the empirical L-R relation (Wandel, Peterson \& Malkan 1999; (Kaspi et al. 2000). We note that the timescale of the SF-break is strongly correlated with the black hole mass, the best fit being consistent with a linear relation, $M_{\mathrm{BH}}=$ $1.010^{6}\left(\tau_{S F} /\right.$ day $)$. Almost all points coincide, within the errors, with the extrapolated "CygX1 line" marked by the dashed diagonal lines in fig. 3, which shows the extrapolation from the break time - $M_{\mathrm{BH}}$ relation for the two

breaks in the PSD of Cyg X-1, corresponding to $0.2 \mathrm{sec}$ and $5 \mathrm{sec}$, respectively. This resultsis consistent with similar trend found in AGN between the black hole mass and the break time in the PSD.

We acknowledge a long lasting collaboration with Alex Markowitz. AW acknowledges the hospitality of the Physics and Astronomy department at UCLA.

\section{References}

Chatterjee et al. 2009, ApJ 704, 1689

Chatterjee et al. 2011, ApJ 734, 43

Collier, S. ES Peterson, B. 2001, ApJ 2001,555, 775

Edelson, R. E Nandra, K. 1999, ApJ 514, 682

Emmanoulopoulos, D., ES McHardy, I. M. and Uttley, P. 2010, MNRAS 404, 931

Kaspi, S. et al. 2000, ApJ 533, 631

Kataoka et al. . 2001, ApJ 560, 659

Kelly, B. C. et al. 2014, ApJ 788, 33

Markowitz et al. 2003, ApJ 593, 96

McHardy et al. 2005, MNRAS 348, 783

McHardy, I. M. et al. 2006, Nature 444730

Peterson, B. et al. 2004, ApJ 613, 632

Rothschild, R. E., et al. 2011, ApJ 733, 23

Simonetti et al.. 1985, ApJ 295, 46S

Timmer \& Koenig 1995, A \& A300, 707

Uttley,P., McHardy, I. M. ES Papadakis 2002, MNRAS 332,231

Uttley, P. \& McHardy, I. M. 2005, MNRAS 363,586

Wandel, A. \& Mushotzky, R. F. 1986, ApJ 306, L61

Wandel, A., Peterson, B.EJ Malkan, M. 1999, ApJ 526, 579

Webb, W.EG Malkan, M. 2000, ApJ 540,652 